Old Dominion University

ODU Digital Commons

Physics Faculty Publications

Physics

6-1992

\title{
Direct Measurement of the Van Der Waals Interaction Between an Atom and Its Images in a Micron-Sized Cavity
}

\author{
V. Sandoghdar \\ C. I. Sukenik \\ Old Dominion University, csukenik@odu.edu
}

E. A. Hinds

S. Haroche

Follow this and additional works at: https://digitalcommons.odu.edu/physics_fac_pubs

Part of the Atomic, Molecular and Optical Physics Commons

\section{Repository Citation}

Sandoghdar, V.; Sukenik, C. I.; Hinds, E. A.; and Haroche, S., "Direct Measurement of the Van Der Waals Interaction Between an Atom and Its Images in a Micron-Sized Cavity" (1992). Physics Faculty Publications. 6.

https://digitalcommons.odu.edu/physics_fac_pubs/6

\section{Original Publication Citation}

Sandoghdar, V., Sukenik, C.I., Hinds, E.A., \& Haroche, S. (1992). Direct measurement of the van der Waals interaction between an atom and its images in a micron-sized cavity. Physical Review Letters, 68(23), 3432-3435. doi: 10.1103/PhysRevLett.68.3432

This Article is brought to you for free and open access by the Physics at ODU Digital Commons. It has been accepted for inclusion in Physics Faculty Publications by an authorized administrator of ODU Digital Commons. For more information, please contact digitalcommons@odu.edu. 


\title{
Direct Measurement of the van der Waals Interaction between an Atom and Its Images in a Micron-Sized Cavity
}

\author{
V. Sandoghdar, C. I. Sukenik, and E. A. Hinds \\ Physics Department, Yale University, New Haven, Connecticut 06520 \\ Serge Haroche \\ Ecole Normale Supérieure, Paris, France and Physics Department, Yale University, New Haven, Connecticut 06520
}

(Received 27 March 1992)

\begin{abstract}
We have measured by laser spectroscopy the energy of interaction between a sodium atom and its images in the walls of a micron-sized cavity. This cavity-QED study is the first direct quantitative test of the Lennard-Jones van der Waals interaction as a function of controlled atom-surface separation and mean-square electric dipole moment.
\end{abstract}

PACS numbers: $42.50 . \mathrm{Wm}, 32.70 . \mathrm{Jz}$

The van der Waals interaction [1] between extended neutral bodies is a ubiquitous feature of nature in which a cooperative electrical polarization of the bodies lowers the energy of the system. The most familiar case is that of a gas in which the fluctuating dipole of each molecule polarizes its neighbors [2], resulting in an attractive potential proportional to $R^{-6}$, where $R$ is the distance between two molecules. This interaction is responsible for a famous correction to the ideal-gas equation, causes shifts and broadening of spectral lines in vapors, and plays an essential role in the latent heat and surface tension of liquids. Another important example is the Lennard-Jones [3] dipole attraction between an atom and its image in a plane conductor, for which the potential varies as $z^{-3}$ where $z$ is the distance of the atom from the mirror. This atom-surface coupling is involved in a variety of physicochemical processes, such as adsorption, in which atoms come close to surfaces. Atomic-force microscopes are also based on the van der Waals interaction [4].

Although several direct, quantitative measurements have been made of the collective van der Waals forces between macroscopic bodies [5], experimental studies of the van der Waals interaction between a free atom or molecule and a macroscopic surface are scarce. Some experiments have been based on the observation of atomic trajectories near metallic surfaces [6] where a small atomic deflection, related to the cumulative effect of van der Waals interactions at various distances, yielded qualitative information on the interaction itself. A small frequency shift, recently observed in the spectrum of cesium vapor near a dielectric surface [7], has also been related to the van der Waals atom-surface interaction integrated over a range of separations. In this Letter we report the first direct and precise measurement of the van der Waals coupling between an atom and a metallic surface, in which the interaction is quantitatively studied as a function of controlled separation and of the electronic state of the atom.

Figure 1 (a) shows the main features of the experiment. An atomic beam of sodium effuses from an oven at $200^{\circ} \mathrm{C}$ into a $10^{-7}$-torr vacuum and enters the space between two plane, gold-coated mirrors (flatness $\sim 5 \mathrm{~nm}$ ), which is $8 \mathrm{~mm}$ long in the beam direction and $3 \mathrm{~cm}$ high. Approximately half a millimeter before the cavity exit, the atoms are excited by two superimposed laser beams, one yellow $(\lambda=589 \mathrm{~nm})$ and the other blue $(\lambda \sim 425 \mathrm{~nm})$, either to the $10 D_{5 / 2}$ state or to one of the states $n S$, with $n=10-13$. The relevant energy levels are shown in Fig. 1 (b). The laser beams are focused to a $300 \mu \mathrm{m}$ waist and are carefully aligned to be normal to the mirrors, and therefore to the atomic beam, in order to avoid any firstorder Doppler effect. Excited atoms leave the cavity and enter a detector where they are field ionized and counted using a channel electron multiplier. An excitation spectrum is obtained by scanning the wavelength $\lambda_{2}$ while

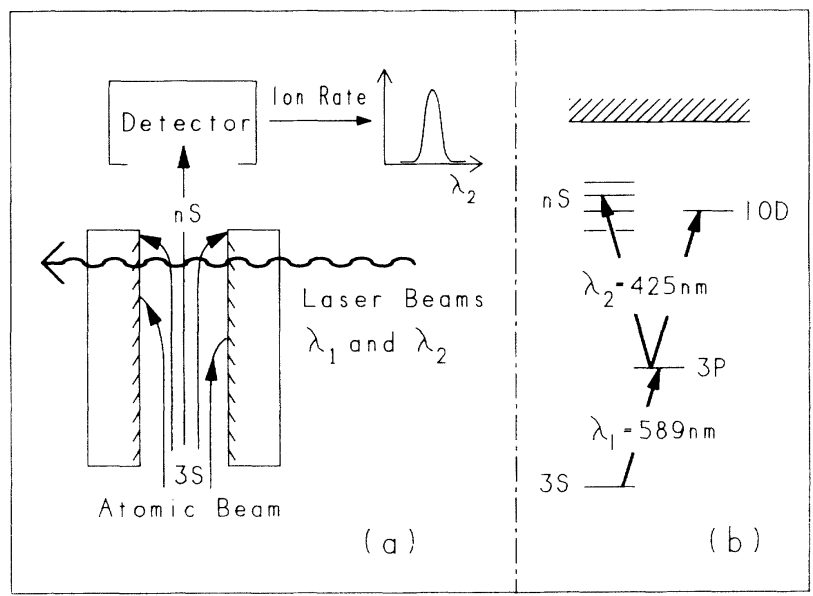

FIG. 1. (a) Sodium ground-state atomic beam enters the parallel-plate cavity and is excited by laser beams with wavelengths $\lambda_{1}=589 \mathrm{~nm}$ and $\lambda_{2} \sim 425 \mathrm{~nm}$ to an upper level $n S$ $(n=10-13)$ or $10 D_{5 / 2}$. The wavelength $\lambda_{2}$ is scanned to obtain an excitation spectrum. Only those atoms excited near the center of the cavity reach the detector. (b) Relevant energy levels of sodium. 
recording the rate of detected ions. The essence of the experiment is to study the shifts of the $n S$ excitation spectra at various mirror spacings, the $10 D_{5 / 2}$ spectra being used to monitor stray electric fields as discussed below.

Figure 2 shows the van der Waals potential for the $13 S$ state of sodium in a parallel-plate cavity $1 \mu \mathrm{m}$ wide due to the infinite series of images resulting from successive field reflections. The interaction energy is negative and diverges at each mirror surface. At the center of the gap, this potential reaches a maximum having the value $[8,9]$

$$
h \delta_{\mathrm{vdW}}=\frac{-7 \zeta(3)}{24 \pi \varepsilon_{0} L^{3}}\left\langle n S\left|d^{2}\right| n S\right\rangle,
$$

where $\zeta(3) \cong 1.20$ is the Riemann zeta function, $d$ is the electric dipole operator, and $L$ is the width of the gap. We have computed the expectation values of $d^{2}$ using the known oscillator strengths between $n S$ and $n^{\prime} P$ states [10] and find that the result is given to better than $1 \%$ accuracy by the hydrogenic formula $\left\langle n S\left|d^{2}\right| n S\right\rangle$ $=e^{2} a_{0}^{2} n^{* 2}\left(5 n^{* 2}+1\right) / 2$, where $e$ is the electron charge, $a_{0}$ is the Bohr radius, and $n^{*}=n-1.349$ is the principal quantum number corrected for the quantum defect of the $S$ states.

At distances of the order of atomic dimensions, higher-multipole interactions and electron exchange effects become important and the atom-surface interaction no longer has the van der Waals form. For atommirror separations greater than $(2 \pi)^{-1}$ times the characteristic atomic absorption or emission wavelengths, the propagation time of the field exceeds the autocorrelation time of the atomic dipole, and the van der Waals interaction vanishes. It is replaced in ground states by the Casimir-Polder interaction [11], which varies as $z^{-4}$ and is a purely quantum electrodynamic effect. The longrange behavior for excited states, which is an oscillatory function of the separation, is dominated instead by the resonant radiative coupling between the atom and its re-

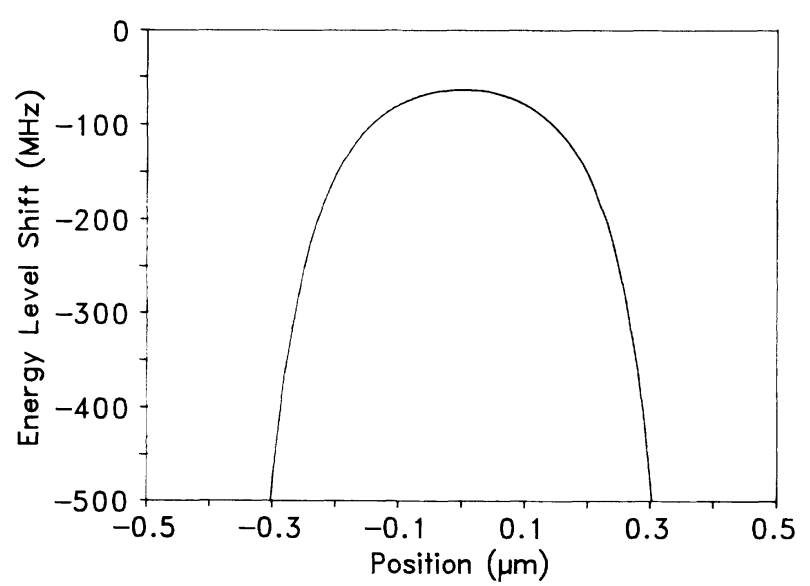

FIG. 2. van der Waals shift of the sodium $13 S$ state vs position in a $1-\mu \mathrm{m}$-wide, parallel-plate cavity. tarded reflections [8]. The $n S$ states of this study have an electronic extension of order $10 \mathrm{~nm}$ and their characteristic emission and absorption wavelengths $\left(n S \rightarrow n^{\prime} P\right)$ are in the 50-150 $\mu \mathrm{m}$ range. Thus the van der Waals formula (1) holds for $L$ between $\sim 100$ and $\sim 10000 \mathrm{~nm}$ and is valid for the full range of gap sizes we have explored. Moreover, our spectra are sensitive only to the shifts of the excited $n S$ levels since the shifts of the $3 S$ and $3 P$ states are completely negligible in comparison.

At first sight, the potential shown in Fig. 2 suggests very asymmetric spectral lines with a high-frequency edge due to atoms at the center of the gap and a long tail on the low-frequency side from atoms passing closer to either mirror. In fact, the lines are typically only 20-30 $\mathrm{MHz}$ wide, indicating that the atoms we detect are excited only near the center of the gap over a region of width $0.2 L-0.3 L$. This is due to a combination of several effects. First, before laser excitation, atoms far from the center are deflected by the atom-cavity interaction onto the mirrors where they stick and are therefore removed from the beam. Second, the excited atoms experience a much larger attraction to the walls and cannot escape from the cavity to be detected unless they are sufficiently close to the center at the point of laser excitation. Finally, the detected atoms contribute to the spectrum in proportion to the inverse of the slope of the potential at their position, which again favors those excited at the center.

We now give some more details about the experimental procedure. The mirror surfaces are made by thermal evaporation of chromium $(\sim 1 \mathrm{~nm})$ followed by gold $(40$ $\mathrm{nm}$ ) onto a substrate of fused silica. This results in modest reflectivities at the laser wavelengths $(67 \%$ at $589 \mathrm{~nm}$ and $32 \%$ at $425 \mathrm{~nm}$ ) and allows us to couple light into the cavity even though it is not resonant at these wavelengths. On the other hand, the reflectivity of the gold film is essentially unity at the much longer wavelengths (50-150 $\mu \mathrm{m})$ relevant for the van der Waals interaction of the $n S$ states. The mirrors are pressed against each other to form a wedge whose width is zero at the bottom, while the width at the top can be varied by pushing on the mirrors with screws in order to compress a 3- $\mu \mathrm{m}$ nickel foil spacer acting as a spring. We set the width of the gap by illuminating the cavity with monochromatic light (mercury green, $\lambda=546 \mathrm{~nm}$ ) and adjusting the screws until one of the interference fringes coincides with the laser beams.

Excitation spectra were observed for four $n S$ states ( $n=10-13)$ for each of the seven cavity widths $L$ corresponding to the third through ninth fringes of the green light. In Fig. 3, graphs (a)-(e) show some of the $13 S$ spectra in successively narrower cavities. The peak on the right of each trace is a reference line obtained on unperturbed atoms in an auxiliary atomic beam. Looking at the peak on the left, the van der Waals shift is clearly seen.

Three main systematic effects must be taken into account when analyzing these spectra. First, in spite of the strong spatial selection of the atomic beam, the peaks of 


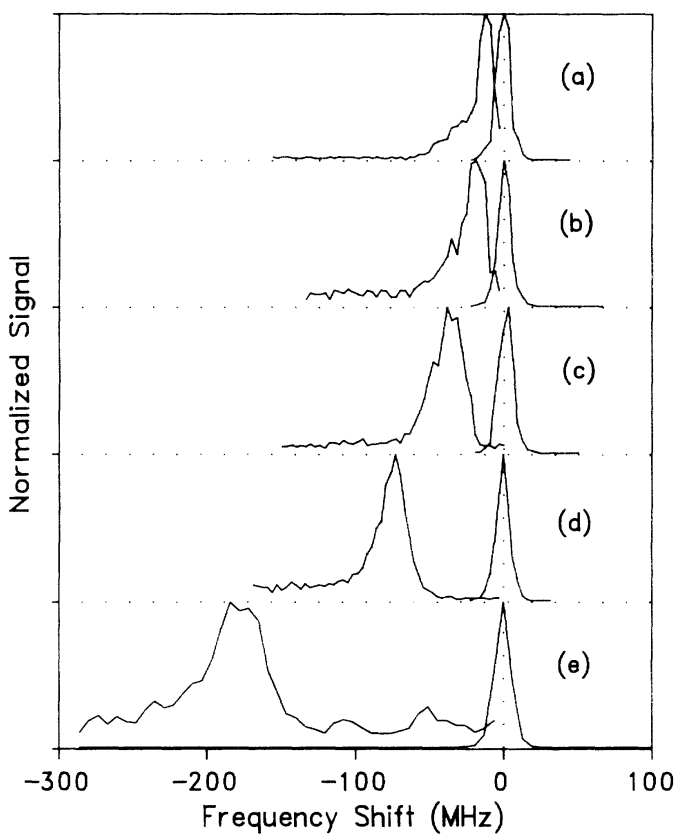

FIG. 3. Shifted excitation spectra of the sodium $13 S$ state in a cavity. Traces (a)-(e) were measured in cavities of widths $2100,1554,1281,1007$, and $734 \mathrm{~nm}( \pm 21 \mathrm{~nm})$, respectively.

the spectral lines lie at a slightly lower frequency than they would if all the atoms were exactly at the center of the cavity. A simple numerical model shows that this offset $\delta_{\text {offset }}$ grows with linewidth and reaches a maximum of $-3.3 \mathrm{MHz}$ when the observed linewidth is larger than $1.5 \Gamma, \Gamma$ being the free space linewidth which is $11 \mathrm{MHz}$ in our experiment.

A second small correction is required because of the Stark shift $\delta_{\text {Stark }}$ of the $n S$ states due to an unavoidable stray electric field in the cavity. This electric field produces much larger Stark shifts of the very polarizable $10 D_{5 / 2, m_{J}}=5 / 2$ sublevel, which we measure to determine the required correction. In order to find the $n S$ Stark shift $\delta_{\text {Stark, we subtract the calculated van der Waals shift }}$ of the $10 D_{5 / 2, m_{j}}=5 / 2$ level from the measured shift of that level and divide by the ratio of electric polarizabilities, $\alpha\left(10 D_{5 / 2, m_{j}=_{5 / 2}}\right) / \alpha(n S)$. This ratio is calculated to vary from 24 for $n=13$ to 150 for $n=10$, a result that we checked by recording Stark spectra in known electric fields outside the cavity. We note that due to the large values of these ratios, the Stark correction is actually very insensitive to the assumed value of the $10 D_{5 / 2, m_{J}}=5 / 2$ van der Waals shift. The largest $n S$ Stark shift is found in the $13 S$ state and in the smallest gap, where $\delta_{\text {meas }}$ $=-178 \mathrm{MHz}$ while $\delta_{\text {Stark }}=-6 \mathrm{MHz}$, corresponding to a stray field of $44 \mathrm{~V} / \mathrm{cm}$. In every case the correction is less than $10 \%$ of the van der Waals shifts observed. In order to reach this low level of stray electric field, we find it important to bake the mirrors at $120^{\circ} \mathrm{C}$ for several hours after placing them in the vacuum system. The

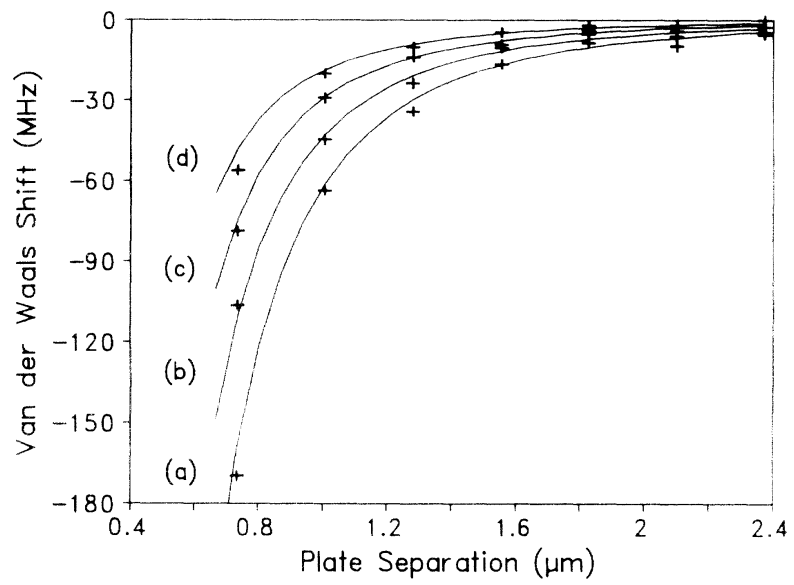

FIG. 4. van der Waals shifts vs plate separation for an atom at cavity center. Series $a-d$ correspond to states $13 S-10 S$, respectively, with crosses indicating experimental data and lines corresponding to the calculated shifts.

electric field is then found to vary roughly as $L^{-2}$, which is consistent with a patch effect due to the crystalline structure of the evaporated gold surface.

The last systematic correction concerns our measurement of the cavity width using the green light fringes. If the phase of the reflection coefficient is written as $\pi+\phi$, the width of the cavity at the $m$ th fringe is $(m-\phi / \pi) \lambda / 2$. When $\phi$ is estimated using $0.32+2.22 i$ for the complex refractive index of gold at $546 \mathrm{~nm}$, we find that the cavity should be narrower than $m \lambda / 2$ by $72 \mathrm{~nm}$. Direct measurements on a similar cavity using an electron microscope show that the actual correction is $85 \pm 15 \mathrm{~nm}$.

The crosses in Fig. 4 show the observed van der Waals shifts, $\delta_{\text {meas }}-\delta_{\text {offset }}-\delta_{\text {Stark, versus gap width for each of }}$ the four $n S$ levels. The width of the cross $( \pm 21 \mathrm{~nm})$ reflects the total uncertainty in the mirror spacing, while the height indicates a $\pm 2 \mathrm{MHz}$ uncertainty in frequency shift due to residual Doppler shift and measurement errors. The lines correspond to Eq. (1), without any free parameters.

This experiment provides the first direct and precise check of the Lennard-Jones formula. The result clearly verifies both the $z^{-3}$ law and the proportionality to the mean-square atomic dipole. We see that the boundary around the atom affects not only the natural widths of the levels, as was demonstrated in earlier cavity-QED studies [9], but also shifts the energy levels by an amount which, in the distance range of our experiment, is given precisely by the Lennard-Jones theory. This shift results from the near-field instantaneous coupling of the atomic dipole with its images in the mirrors. In the case of larger mirror spacings, one expects the excited state to exhibit instead the oscillatory shift due to the far-field retarded radiative coupling of the atom with its images [8], which is proportional to $1 / n^{* 2} L$, rather than $n^{* 4} / L^{3}$. This work may open the way to observing the far-field shift in a 
low- $Q$ cavity, where its magnitude is typically a fraction of the natural width of the state. This radiative shift has been observed in high- $Q$ cavities resonant near an atomic transition frequency, where it is strongly enhanced [12]. The retarded Casimir shift of the ground state has so far only been investigated by the deflection or reflection of an atomic beam [13]. Since any transition to an excited level is affected by the much larger shift and linewidth of that level, a spectroscopic study of the Casimir shift seems inadequate, but methods based on atom interferometry offer an interesting alternative.

We have shown that it is practical to perform spectroscopy on an atomic beam inside a cavity less than $1 \mu \mathrm{m}$ wide, opening the way to direct studies of other effects including the patch potentials of a surface and the excitedstate atom-surface coupling. From another point of view, this experiment shows that the van der Waals energy shift can be used to determine the position of an atom within a microscopic cavity with a resolution of a few tens of nanometers, a new tool which also promises to be valuable in future cavity-QED studies.

We thank Donghyun Cho for his work on the dataacquisition system. This work was supported by the $\mathrm{Na}$ tional Science Foundation.

[1] See, for example, D. Langbein, Springer Tracts in
Modern Physics, Vol. 72 (Springer-Verlag, New York, 1972).

[2] F. London, Z. Phys. 63, 245 (1930); Trans. Faraday Soc. 33N, 8 (1937).

[3] J. E. Lennard-Jones, Trans. Faraday Soc. 28, 333 (1932).

[4] G. Binnig, C. F. Quate, and Ch. Gerber, Phys. Rev. Lett. 56, 930 (1986).

[5] See, for example, W. Arnold, S. Hunklinger, and K. Dransfeld, Phys. Rev. B 19, 6049 (1979), and references therein.

[6] D. Raskin and P. Kusch, Phys. Rev. 179, 712 (1969); A. Shih, D. Raskin, and P. Kusch, Phys. Rev. A 9, 652 (1974); J. Bardon and M. Audiffren, J. Phys. (Paris), Lett. 44, 883 (1983); A. Anderson et al., Phys. Rev. A 37, 3594 (1988).

[7] M. Oria et al., Europhys. Lett. 14, 527 (1991).

[8] G. Barton, Proc. R. Soc. London A 410, 175 (1987); E. A. Hinds and V. Sandoghdar, Phys. Rev. A 43, 398 (1991).

[9] E. A. Hinds, Adv. At. Mol. Opt. Phys. 28, 237 (1991).

[10] E. M. Anderson and V. A. Zilitis, Opt. Spectrosc. (USSR) 16, 99 (1964).

[11] H. B. Casimir and D. Polder, Phys. Rev. 73, 360 (1948).

[12] D. J. Heinzen and M. S. Feld, Phys. Rev. Lett. 59, 2623 (1987); Yifu Zhu et al., Phys. Rev. Lett. 64, 2499 (1990); F. Bernardot et al., Europhys. Lett. 17, 33 (1992); R. J. Thompson et al., Phys. Rev. Lett. 68, 1132 (1992).

[13] E. A. Hinds et al., in Atomic Physics, Vol. 12, edited by J. Zorn and R. Lewis (AIP, New York, 1991), p. 283; Mark Kasevich et al., ibid., p. 47. 